associated with Fig. 1. We can see that, unless the birds fly closer together than observations suggest, the aerodynamic effects of such flight in two-dimensional flocks is slight; even if the spacing between birds is as little as twice the wingspan $(\Delta \sim 2 d)$, the uplift effect in a horizontally extended flock is only around $10 \%$. Higdon and Corrsin estimate the corrections to equation (1), in the event of the birds flying in some ordered lattice pattern, rather than more-or-less randomly. These correction terms are of order $n^{2} d^{4}$; that is, they are smaller than those given in equation (1), by a factor of order $(d / \Delta)^{2}$.

Extending the analysis to encompass a fully three-dimensional flock is in principle straightforward (by virtue, again, of Munk's stagger theorem, which enables the three-dimensional problem to be projected into a two-dimensional one). In a randomly arranged flock, the upshot will be to regain the expression (1), but with $n$ now being the average number of birds projected onto unit area perpendicular to the flight direction. Clearly, the result will be to increase the uplift effect (1) as $n$ increases, provided the flock is extended more horizontally than vertically. If the effective value of $\boldsymbol{n}$ becomes too large (so that $n d^{2}$ is not appreciably less than unity), the 'dipole vortex' approximation will begin to break down, and the computations must be based on pairs of vortices centred at a bird's two wingtips. But for most three-dimensional flocks of migratory birds the density is unlikely to make this refinement necessary. I take home the message that three-dimensional flocks of passerines and other birds can gain non-negligible updraft effects, provided the flock is extended more horizontally than vertically, but that the magnitude of the effect is not nearly as large as that gained in the close, horizontal line formation of geese.

A different kind of insight into the dynamics and ergonomics of migratory flight comes from Allerstam ( $J$. theor. Biol. 79, 341; 1979). Starting with the observation that wind speed is usually less at low than at high altitude, he examines the idea that migratory birds may save flying time and energy by allowing themselves partly to be drifted by strong winds at high altitude, and then correcting their course at low altitude in relatively weaker winds. For typical values of wind velocities, Allerstam's calculations suggest this behaviour will be advantageous if the strong upper winds are blowing in a direction roughly $30^{\circ}$ to $90^{\circ}$ wide of the goal direction ('following side winds'). If the wind is blowing at an angle less than $30^{\circ}$ to the desired direction, a direct high altitude flight is optimal. Conversely, a direct low altitude flight is the best route against a headwind (in excess of $90^{\circ}$ wide of the goal direction). Allerstam concludes with a survey of the available evidence, of which the best (Gruys-Casimir, Arch.
Neerl. Zool. 16, 175; 1965) is on chaffinch migration in Holland: "Radar observations of drift in high altitude bird migration and visual records of low altitude overcompensation are compatible with the optimal flight behaviour of migrants at high and low altitude, respectively, as predicted from this hypothesis."

\section{Mycorrhizal associations}

\section{from Peter D. Moore}

MYCORRHIZAL associations between plant roots and fungi are now known to be extremely common in nature. It is evident that such associations are symbiotic, both parties benefitting from the interaction. As far as the plant is concerned, the association may be very costly in terms of the loss of fixed carbon to its heterotrophic partner. Lewis and Harley (New Phytol. $64,256 ; 1965)$ fed ${ }^{14} \mathrm{C}$ sucrose to cut roots of beech (Fagus sylvatica) and observed that $50-75 \%$ of the carbon reaching the root tip was passed on to the mycrorrhizal fungus, which obviously represents a considerable drain on the photosynthetic resources of the plant.

The widespread occurrence of mycorrhizae, however, witnesses to the evolutionary value of the partnership to host plant as well as fungus. It has long been established that a major advantage to the plant is the greater efficiency with which it is able to absorb necessary inorganic materials from the soil (Harley $J$. Ecol. 59, 653; 1971). There is also some evidence to suggest that water uptake is assisted by mycorrhizal infection (Safir $e t$ al. Plant Physiol. 49, 700; 1972). This being so, one might expect mycorrhizal associations to be of particular value, and therefore especially prevalent, in habitats deficient in plant nutrients and water.

Grime (Plant Strategies and Vegetation Processes, 36; Wiley, Chichester, New York, 1979) takes up this argument in his discussion of stress-tolerant plant species. Grime proposes an ecological classification of plants into competitors, stresstolerators and ruderals, and he claims that mycorrhizal associations, particularly of an ectotrophic type, are to be expected amongst plant species adapated to nutrient stresses. Data recently published deriving from arid regions of the United States support and extend this view.

Reeves, Wagner, Moorman and Kiel (Am. J. Bot. 66, 6; 1979) have conducted a survey of vesicular-arbuscular (endo-) mycorrhizae in semi-arid sagebrush communities of Colorado. Of the 42 species of plant present, only 2 were found

Peter D. Moore is a Senior Lecturer in the Department of Plant Sciences, King's College, London. to lack mycorrhizal infection, one chenopod and one crucifer. This emphasises the widespread nature of mycorrhizae in a taxonomically diverse flora: 13 families are represented and all but one have mycorrhizal members present.

The authors also investigated a nearby site which had been severely disturbed by road construction. Here 21 plant species were growing, but only 6 possessed mycorrhizal infections; these species had also been present in the undisturbed site. The remaining 15 species were confined to the region of disturbance and had no detectable mycorrhizal infection (three individuals of each species were examined). Thus, in an unstable habitat, the mycorrhizal plants could be at a disadvantage, perhaps because of the destruction of a large proportion of the residual fungus in the soil during the process of disturbance which might restrict recolonisation by mycorrhizal species of plants. The authors propose that early successional stages are deficient in mycorrhizal species, whereas in later, more stable stages the vast majority of species are so adapted. This seems to be justified for their arid, nutrient-poor habitat.

The lack of mycorrhizal species in disturbed sites is confirmed by the results of a similar investigation by Miller (Can. J. Bot. 57, 619; 1979) of mine spoil heaps and nearby undisturbed sage scrub in Wyoming. In these latter, pristine areas, 21 species were examined and only one lacked vesicular-arbuscular mycorrhizal infection. On the spoil heaps 16 species were found, none of which was infected. Even within one family, the Chenopodiaceae, some members are nonmycorrhizal and ruderal in habit, whereas others (the majority) are mycorrhizal and are found in undisturbed habitats where edaphic stresses of nutrient and water availability are encountered.

So the Grime hypothesis seems to hold true for these semi-arid ecosystems: there does appear to be a division among plants which corresponds to his stress-tolerant and ruderal types. What is not clear from these particular sites is the outcome of low disturbance and low stress. In other words, of what importance are endomycorrhizae to the Grime competitor?

\section{0 years ago}

M.A. Guyard claims to have discovered another new metal of the platinum group which he names uralium, from the Ural Mountains, whence the ore is procured. There have been quite a flood of similar announcements lately. We have now gallium, davyum, mosandrum, neptunium, decipium, phillipium, norvegium, scandium, ytterbium, holmium, " $X$ ", thulium, and uralium. Chemists will have to keep as narrow a watch on these minor elements as our astronomers do upon the minor planets, or we shall not know where we are.

From Nature 21, 25 Dec., 187; 1879. 